\title{
Inclisiran for the Treatment of Heterozygous Familial Hypercholesterolemia
}

\author{
Frederick J. Raal, M.D., Ph.D., David Kallend, M.B., B.S., \\ Kausik K. Ray, M.D., M.Phil., Traci Turner, M.D., Wolfgang Koenig, M.D., \\ R. Scott Wright, M.D., Peter L.J. Wijngaard, Ph.D., Danielle Curcio, M.B.A., \\ Mark J. Jaros, Ph.D., Lawrence A. Leiter, M.D., and John J.P. Kastelein, M.D., Ph.D., \\ for the ORION-9 Investigators*
}

From the Faculty of Health Sciences, University of the Witwatersrand, Johannesburg, South Africa (F.J.R.); the Medicines Company, Zurich, Switzerland (D.K.); the Imperial Centre for Cardiovascular Disease Prevention, Department of Primary Care and Public Health, Imperial College, London (K.K.R.); Medpace Reference Laboratories, Cincinnati (T.T.); Deutsches Herzzentrum München, Technische Universität München, and German Center for Cardiovascular Research, Munich Heart Alliance, Munich (W.K.), and the Institute of Epidemiology and Medical Biometry, University of Ulm, Ulm (W.K.) - all in Germany; the Division of Preventive Cardiology and the Department of Cardiology, Mayo Clinic, Rochester, MN (R.S.W.); the Medicines Company, Parsippany, NJ (P.L.J.W., D.C.); Summit Analytical, Denver (M.J.J.); Li Ka Shing Knowledge Institute, St. Michael's Hospital, University of Toronto, Toronto (L.A.L.); and the Department of Vascular Medicine, Academic Medical Center, University of Amsterdam, Amsterdam (J.J.P.K.) Address reprint requests to Dr. Raal at the Division of Endocrinology and Metabolism, Faculty of Health Sciences, University of the Witwatersrand, 7 York Rd., Parktown, Johannesburg 2193, South Africa, or at frederick.raal@wits.ac.za.

*A list of the ORION-9 investigators is provided in the Supplementary Appendix, available at NEJM.org.

This article was published on March 18, 2020, at NEJM.org.

N Engl J Med 2020;382:1520-30 DOI: 10.1056/NEJMoal913805

Copyright (c) 2020 Massachusetts Medical Society.
ABSTRACT

\section{BACKGROUND}

Familial hypercholesterolemia is characterized by an elevated level of low-density lipoprotein (LDL) cholesterol and an increased risk of premature atherosclerotic cardiovascular disease. Monoclonal antibodies directed against proprotein convertase subtilisin-kexin type 9 (PCSK9) have been shown to reduce LDL cholesterol levels by more than $50 \%$ but require administration every 2 to 4 weeks. In a phase 2 trial, a twice-yearly injection of inclisiran, a small interfering RNA, was shown to inhibit hepatic synthesis of PCSK9 in adults with heterozygous familial hypercholesterolemia.

\section{METHODS}

In this phase 3, double-blind trial, we randomly assigned, in a 1:1 ratio, 482 adults who had heterozygous familial hypercholesterolemia to receive subcutaneous injections of inclisiran sodium (at a dose of $300 \mathrm{mg}$ ) or matching placebo on days $1,90,270$, and 450 . The two primary end points were the percent change from baseline in the LDL cholesterol level on day 510 and the time-adjusted percent change from baseline in the LDL cholesterol level between day 90 and day 540.

RESULTS

The median age of the patients was 56 years, and $47 \%$ were men; the mean baseline level of LDL cholesterol was 153 mg per deciliter. At day 510, the percent change in the LDL cholesterol level was a reduction of 39.7\% ( $95 \%$ confidence interval [CI], -43.7 to -35.7 ) in the inclisiran group and an increase of $8.2 \%$ (95\% CI, 4.3 to 12.2 ) in the placebo group, for a between-group difference of -47.9 percentage points (95\% CI, -53.5 to $-42.3 ; \mathrm{P}<0.001$ ). The time-averaged percent change in the LDL cholesterol level between day 90 and day 540 was a reduction of $38.1 \%$ (95\% CI, -41.1 to -35.1$)$ in the inclisiran group and an increase of $6.2 \%$ (95\% CI, 3.3 to 9.2) in the placebo group, for a between-group difference of -44.3 percentage points (95\% CI, -48.5 to -40.1 ; $\mathrm{P}<0.001$ ). There were robust reductions in LDL cholesterol levels in all genotypes of familial hypercholesterolemia. Adverse events and serious adverse events were similar in the two groups.

\section{CONCLUSIONS}

Among adults with heterozygous familial hypercholesterolemia, those who received inclisiran had significantly lower levels of LDL cholesterol than those who received placebo, with an infrequent dosing regimen and an acceptable safety profile. (Funded by the Medicines Company; ORION-9 ClinicalTrials.gov number, NCT03397121.) 
$\mathrm{H}$ ETEROZYGOUS FAMILIAL HYPERCHOlesterolemia, a genetic disorder that affects 1 in 250 persons or 30 million people worldwide, is characterized by elevated levels of low-density lipoprotein (LDL) cholesterol from birth. Without treatment, the condition is associated with premature complications and death from accelerated development of atherosclerotic cardiovascular disease. ${ }^{1}$

Variants in the gene encoding the LDL receptor (LDLR) account for more than $90 \%$ of cases of familial hypercholesterolemia, whereas variants in other genes, such as those encoding apolipoprotein $\mathrm{B}(\mathrm{APOB})$ and proprotein convertase subtilisinkexin type 9 (PCSK9), account for $5 \%$ and less than $2 \%$ of cases, respectively. ${ }^{2}$ However, despite the use of next-generation sequencing, a monogenic variant cannot be identified in up to $30 \%$ of patients who have received a clinical diagnosis of definite heterozygous familial hypercholesterolemia. ${ }^{3}$ Since the risk of atherosclerotic cardiovascular disease is driven by the degree and duration of an elevated LDL cholesterol level, the goal of management should be the initiation of therapy to lower the LDL cholesterol level as soon as possible after diagnosis, with even more intensive lipid-lowering therapy in patients with established atherosclerosis. ${ }^{4}$

Pharmacologic management of familial hypercholesterolemia includes the use of high-intensity statins, ezetimibe, and monoclonal antibodies directed against circulating PCSK9. Monoclonal antibodies against PCSK9 have been shown to reduce LDL cholesterol levels by more than $50 \%$ but require administration every 2 to 4 weeks. ${ }^{5,6}$ The phase 2 ORION-1 trial showed that inclisiran, a small interfering RNA targeting hepatic PCSK9 synthesis, has the potential to substantially reduce LDL cholesterol levels with an acceptable sideeffect profile and an infrequent dosing regimen. ${ }^{7}$ Here, we report the results of the phase 3 ORION-9 trial, in which we evaluated the use of inclisiran in a large cohort of adult patients with heterozygous familial hypercholesterolemia who had been treated with a maximally accepted dose of statin therapy.

METHODS

TRIAL OVERSIGHT AND DESIGN

ORION-9 was a double-blind, randomized, placebo-controlled trial that was conducted in 8 countries at 46 sites. The trial protocol (available with the full text of this article at NEJM.org) was approved by an institutional review board or independent ethics committee at each participating institution. All the trial patients provided written informed consent. The trial was designed by the academic steering committee and the sponsor, the Medicines Company. The first author wrote the initial draft of the manuscript, and all the authors had access to the data and contributed to the review of and revisions to the initial draft of the manuscript. All the authors vouch for the accuracy and completeness of the data and for the fidelity of the trial to the protocol.

\section{PATIENTS}

The diagnosis of familial hypercholesterolemia was based on genetic confirmation or established phenotypic Simon Broome criteria. ${ }^{8}$ The patients were required to have an LDL cholesterol level of at least $100 \mathrm{mg}$ per deciliter (2.6 mmol per liter) despite receiving a maximally accepted dose of statin therapy with or without ezetimibe. Patients who were receiving a PCSK9 monoclonal antibody were excluded. Details regarding the inclusion and exclusion criteria are provided in the Supplementary Appendix, available at NEJM.org.

\section{TRIAL PROCEDURES}

The patients were randomly assigned in a 1:1 ratio to receive inclisiran sodium (at a dose of $300 \mathrm{mg}$, which corresponds to a dose of $284 \mathrm{mg}$ of inclisiran free acid) or matching placebo, which were both administered as a 1.5-ml subcutaneous injection on days $1,90,270$, and 450 . During visits for drug administration, the patients remained under observation for 30 minutes after injection. The patients also attended clinic visits on days 30, 150,330 , and 510 to undergo fasting biochemical measurements and to assess the safety and sideeffect profile of inclisiran. The last trial visit was conducted on day 540 (Fig. S1 in the Supplementary Appendix).

\section{END POINTS}

The two primary end points were the percent change from baseline in the LDL cholesterol level at day 510 and the time-adjusted percent change from baseline in the LDL cholesterol level between day 90 and day 540. Key secondary end points were the mean absolute change from baseline in 
the LDL cholesterol level at day 510 , the timeadjusted absolute reduction from baseline between day 90 and day 540, and changes in levels of PCSK9, total cholesterol, apolipoprotein B, and non-high-density lipoprotein (HDL) cholesterol. Prespecified exploratory end points included the proportion of patients who met the lipid targets for their level of cardiovascular risk and the treatment response according to the underlying genotype of familial hypercholesterolemia.

\section{GENOTYPING}

Next-generation sequencing was performed for the coding regions of the four genes ( $L D L R, A P O B$, PCSK9, and LDLRAP1 [encoding LDLR adaptor protein 1]) that are known to account for the majority of cases of familial hypercholesterolemia. ${ }^{9}$ LDLR exons 1 through 18, APOB exons 1 through 29, PCSK9 exons 1 through 12, and LDLRAP1 exons 1 through 9 were captured, amplified on polymerase-chain-reaction assay, and subjected to pairend DNA sequencing with the use of the Illumina MiSeq sequencing platform. Secondary and tertiary analysis of DNA sequences were performed with the use of the commercial bioinformatics software CLC Genomics Workbench (Qiagen) for variant calling and VarSeq (Golden Helix) for variant analysis. VS-CNV software (Golden Helix) was used to identify DNA copy-number variations in LDLR, which are the cause of familial hypercholesterolemia in up to $10 \%$ of patients. ${ }^{10}$ Variants that were identified were aligned to the GRCh37 (hg19) reference genome, and the pathogenicity of reported variants was determined according to current guidelines. ${ }^{11,12}$ LDLR variants were grouped as pathogenic, probably pathogenic, or of uncertain significance. ${ }^{11,13}$

\section{SAFETY REPORTS}

Adverse events and laboratory values were recorded at all visits through the end-of-trial visit on day 540. Vital signs were recorded at all injection visits and at day 540. Electrocardiography was performed at the time of screening and on days 1 and 540. Investigators classified adverse events as mild, moderate, or severe according to organ class using the criteria of the Medical Dictionary for Regulatory Activities. Injection-site reactions were evaluated with the use of prespecified terms. Antidrug antibodies were measured with the use of a highly sensitive screening method and, if needed, confirmatory assays in accordance with the most recent regulatory guidance.

\section{STATISTICAL ANALYSIS}

The sample size was based on the assumption that the mean $( \pm S D)$ decrease from baseline in the LDL cholesterol level would be at least $30 \pm 20 \mathrm{mg}$ per deciliter $(0.8 \pm 0.5 \mathrm{mmol}$ per liter $)$ more in the inclisiran group than in the placebo group. We calculated that approximately 380 patients would be needed to evaluate efficacy between the inclisiran and placebo groups, assuming a dropout rate of $5 \%$, a two-sided alpha level of 0.05 , and a power of more than $90 \%$ to detect a $30 \%$ reduction from baseline in the LDL cholesterol level. Because of faster-than-expected enrollment, the actual enrollment was 482 patients to allow for all screened and eligible patients to enter the trial.

We used a sequential testing procedure with a two-sided alpha level of 0.05 to assess the percent change in the LDL cholesterol level from baseline to day 510. Once the null hypothesis was rejected, we used a two-sided alpha level of 0.05 to test the time-adjusted percent change from baseline in the LDL cholesterol level between day 90 and day 540. We used a reflexive approach to measuring the LDL cholesterol level in calculating the primary end points. First, we used the Friedewald formula to estimate the LDL cholesterol level. If the LDL cholesterol level was less than $40 \mathrm{mg}$ per deciliter (1.0 mmol per liter) or if the triglyceride levels were more than $400 \mathrm{mg}$ per deciliter (4.5 mmol per liter), we performed preparative ultracentrifugation to directly measure the LDL cholesterol level.

For the first of the two primary end points (the percent change in the LDL cholesterol level from baseline to day 510), we used a multipleimputation washout model to impute missing values. The primary analysis was conducted in the intention-to-treat population and was based on an analysis of covariance (ANCOVA) model on each multiply imputed data set (100 total). The model included the fixed effects of treatment and the baseline LDL cholesterol level. We then used Rubin's model to combine treatment effects from these ANCOVA analyses. For the second primary end point (the time-adjusted percent change in the LDL cholesterol level between day 90 and day 540), we used a control-based pattern-mixture model to impute missing values. We used a mixedeffects model for repeated measurements of data obtained during all visits on each multiply imputed data set. The model included fixed effects for treatment, visit, baseline LDL cholesterol level, and the interaction between treatment and visit. 
Analyses of the secondary end points were performed only after the analyses of the two primary end points were completed and the null hypotheses were rejected. The Hochberg procedure was applied to control for family-wise type I error at a two-sided significance level of 0.05 for the comparison of key secondary end points. We used the two-sided $95 \%$ confidence intervals for least-squares means for testing of continuous variables. Odds ratios and 95\% confidence intervals were used for assessment for binary variables.

We also performed a prespecified subgroup analysis of the percent change from baseline in the LDL cholesterol level according to genotype (the presence or absence of a monogenic familial hypercholesterolemia variant) and according to the presence or absence of variants in LDLR, APOB, and PCSK9. We also determined the mean differences in treatment effect between these subgroups. All analyses were performed with the use of SAS software, version 9.4 (SAS Institute). Details regarding the statistical analysis plans are provided in the protocol.

\section{RESULTS}

\section{TRIAL POPULATION}

The trial was conducted between December 2017 and September 2019. A total of 617 patients were screened; of these patients, 482 underwent randomization (242 patients to receive inclisiran and 240 to receive placebo). In the overall trial population, the mean age was 56 years; 227 patients (47\%) were men, and 453 (94\%) were white (Table 1). Preexisting coronary heart disease was present in $25 \%$ of the patients and diabetes in $10 \%$. The mean baseline LDL cholesterol level was $153.1 \pm 54.0 \mathrm{mg}$ per deciliter $(4.0 \pm 1.4 \mathrm{mmol}$ per liter). A total of $90 \%$ of the patients were receiving statins, including $75 \%$ who were receiving highintensity statins (at least $20 \mathrm{mg}$ of rosuvastatin, $40 \mathrm{mg}$ of atorvastatin, $40 \mathrm{mg}$ of simvastatin, or the equivalent per day); more than $50 \%$ were also receiving ezetimibe. Of the patients in the intention-to-treat population, 235 patients (91.7\%) in the inclisiran group and 231 (96.3\%) in the placebo group completed the trial activities through day 540 (Fig. S2).

\section{PRIMARY END POINTS}

For the first primary end point, the percent change in the LDL cholesterol level from baseline to day 510 was a decrease of $39.7 \%$ ( $95 \%$ confidence in- terval [CI], -43.7 to -35.7 ) in the inclisiran group and an increase of $8.2 \%$ (95\% CI, 4.3 to 12.2) in the placebo group, for a between-group difference of -47.9 percentage points ( $95 \% \mathrm{CI},-53.5$ to -42.3 ; $\mathrm{P}<0.001$ ) (Fig. 1A). For the second primary end point, the time-averaged percent change in the LDL cholesterol level between day 90 and day 540 was a decrease of $38.1 \%$ (95\% CI, -41.1 to -35.1 ) in the inclisiran group and an increase of $6.2 \%$ (95\% CI, 3.3 to 9.2) in the placebo group, for a between group difference of -44.3 percentage points $(95 \% \mathrm{CI},-48.5$ to -40.1 ; $\mathrm{P}<0.001)$. Sensitivity analyses using imputation for missing values produced similar results (Table S1).

\section{KEY SECONDARY END POINTS}

The mean absolute change from baseline in the LDL cholesterol level at day 510 was a decrease of $59.0 \mathrm{mg}$ per deciliter ( $95 \% \mathrm{CI},-64.8$ to -53.2 [1.5 mmol per liter; 95\% CI, -1.7 to -1.4 ]) in the inclisiran group and an increase of $9.9 \mathrm{mg}$ per deciliter (95\% CI, 4.1 to 15.8 [0.3 mmol per liter; $95 \%$ CI, 0.1 to 0.4$]$ ) in the placebo group, for a between-group difference of $-68.9 \mathrm{mg}$ per deciliter $(95 \% \mathrm{CI},-77.1$ to -60.7 [1.8 mmol per liter; $95 \% \mathrm{CI},-2.0$ to -1.6$]$; $\mathrm{P}<0.001$ ) (Fig. 1B). The timeaveraged observed difference in LDL cholesterol levels between day 90 and day 540 was $-56.9 \mathrm{mg}$ per deciliter $(-1.5 \mathrm{mmol}$ per liter) in the inclisiran group and $5.8 \mathrm{mg}$ per deciliter $(0.1 \mathrm{mmol}$ per liter) in the placebo group, for a between-group difference of $-62.6 \mathrm{mg}$ per deciliter $(-1.6 \mathrm{mmol}$ per liter) $(\mathrm{P}<0.001)$, a difference of $44.6 \%$.

At day 510, the percent change in the PCSK9 level was a decrease of $60.7 \%$ (95\% CI, -64.4 to -57.0) in the inclisiran group and an increase of $17.7 \%$ (95\% CI, 13.9 to 21.4 ) in the placebo group, for a between-group difference of -78.4 percentage points $(95 \% \mathrm{CI},-83.7$ to -73.0 ; $\mathrm{P}<0.001)$ (Fig. 1C). At day 510 , the mean absolute change in the PCSK9 level was a decrease of $282.6 \mu \mathrm{g}$ per liter (95\% CI, -297.9 to -267.2 ) in the inclisiran group and an increase of $54.5 \mu \mathrm{g}$ per liter (95\% CI, 39.1 to 70.0) in the placebo group, for a between-group difference of $-337.1 \mu \mathrm{g}$ per liter (95\% CI, -358.9 to $-315.3 ; \mathrm{P}<0.001$ ) (Fig. 1D). The time-averaged observed difference in PCSK9 levels between day 90 and day 540 was $-284.6 \mu$ g per liter (95\% CI, -303.8 to -265.4$)$ in the inclisiran group and $44.0 \mu \mathrm{g}$ per liter (95\% CI, 32.3 to 55.6 ) in the placebo group, for a between group difference of $-328.6 \mu \mathrm{g}$ per liter $(95 \% \mathrm{CI},-351.0$ to -306.1 ; $\mathrm{P}<0.001$ ), a difference of $77 \%$. Waterfall plots for 


\begin{tabular}{|c|c|c|}
\hline Characteristic & $\begin{array}{r}\text { Inclisiran } \\
(\mathrm{N}=242)\end{array}$ & $\begin{array}{l}\text { Placebo } \\
(\mathrm{N}=\mathbf{2 4 0})\end{array}$ \\
\hline Age $(I Q R)-y r$ & $56(47-63)$ & $56(46-64)$ \\
\hline Male sex — no. (\%) & $112(46.3)$ & $115(47.9)$ \\
\hline White race - no. (\%)† & $226(93.4)$ & $227(94.6)$ \\
\hline Atherosclerotic cardiovascular disease — no. (\%) & $59(24.4)$ & $73(30.4)$ \\
\hline \multicolumn{3}{|l|}{ Cardiovascular risk factors — no. (\%) } \\
\hline Current smoker & $28(11.6)$ & $28(11.7)$ \\
\hline Hypertension & $102(42.1)$ & $101(42.1)$ \\
\hline Diabetes & $20(8.3)$ & $28(11.7)$ \\
\hline \multicolumn{3}{|l|}{ Lipid-modifying therapy — no. (\%) } \\
\hline Statin & $219(90.5)$ & $217(90.4)$ \\
\hline High-intensity statin & $185(76.4)$ & $171(71.2)$ \\
\hline Ezetimibe & $135(55.8)$ & $120(50.0)$ \\
\hline \multicolumn{3}{|l|}{ Cholesterol $-\mathrm{mg} / \mathrm{dl}$} \\
\hline Total & $230.0 \pm 54.6$ & $232.4 \pm 62.8$ \\
\hline Low-density lipoprotein & $151.4 \pm 50.4$ & $154.7 \pm 58.0$ \\
\hline High-density lipoprotein & $51.5 \pm 15.1$ & $50.8 \pm 13.1$ \\
\hline Non-high-density lipoprotein & $178.5 \pm 55.4$ & $181.5 \pm 62.5$ \\
\hline Apolipoprotein B - mg/dl & $123.8 \pm 33.2$ & $124.5 \pm 34.8$ \\
\hline Median lipoprotein(a) (IQR) - nmol/liter & $57(22-180)$ & $54(20-185)$ \\
\hline Median triglycerides (IQR) - mg/dl & $120(82-167)$ & $119(85-166)$ \\
\hline Median high-sensitivity C-reactive protein (IQR) - mg/liter & $1.2(0.5-2.9)$ & $1.3(0.6-3.2)$ \\
\hline PCSK9 $-\mu \mathrm{g} /$ liter & $452.2 \pm 131.2$ & $429.1 \pm 135.3$ \\
\hline \multicolumn{3}{|c|}{$\begin{array}{l}\text { Plus-minus values are means } \pm \text { SD. For cholesterol and triglyceride levels, baseline was defined as the value obtained } \\
\text { immediately before the receipt of inclisiran or placebo on trial day } 1 \text {; for other variables, baseline was defined as the } \\
\text { last value before administration of the first dose of inclisiran or placebo. To convert the values for cholesterol to mil- } \\
\text { limoles per liter, multiply by } 0.02586 \text {. To convert the values for triglycerides to millimoles per liter, multiply by } 0.01129 \text {. } \\
\text { IQR denotes interquartile range, and PCSK9 proprotein convertase subtilisin-kexin type } 9 \text {. } \\
\text { Race was reported by the patient. }\end{array}$} \\
\hline
\end{tabular}

individual changes in levels of LDL cholesterol and PCSK9 among the patients are shown in Figure S3.

Inclisiran was associated with lower levels of total cholesterol, non-HDL cholesterol, apolipoprotein $\mathrm{B}$, and triglycerides than placebo, along with higher HDL cholesterol levels. In the inclisiran group, the median level of lipoprotein(a) was reduced by $17.2 \%$ from baseline, but there was little change in the median level of high-sensitivity C-reactive protein (Table S3).

\section{EXPLORATORY OUTCOMES}

At day 510, a reduction from baseline in the mean LDL cholesterol level of $50 \%$ or more was reported in 92 patients $(38.0 \%)$ in the inclisiran group and in $2(0.8 \%)$ in the placebo group $(\mathrm{P}<0.001)$. An LDL cholesterol level of less than $100 \mathrm{mg}$ per deciliter was reported in 158 patients (65.3\%) in the inclisiran group and in 21 (8.8\%) in the placebo group; of these patients, $40.8 \%$ in the inclisiran group and $1.3 \%$ in the placebo group had a level of less than $70 \mathrm{mg}$ per deciliter (1.8 mmol per liter); $19.0 \%$ and $0.8 \%$, respectively, had a level of less than $50 \mathrm{mg}$ per deciliter (1.3 mmol per liter) (Table S2). Among the patients with atherosclerotic cardiovascular disease in the inclisiran group, 38 of 59 (64\%) had an LDL cholesterol level of less than $70 \mathrm{mg}$ per deciliter.

Monogenic familial hypercholesterolemia vari- 


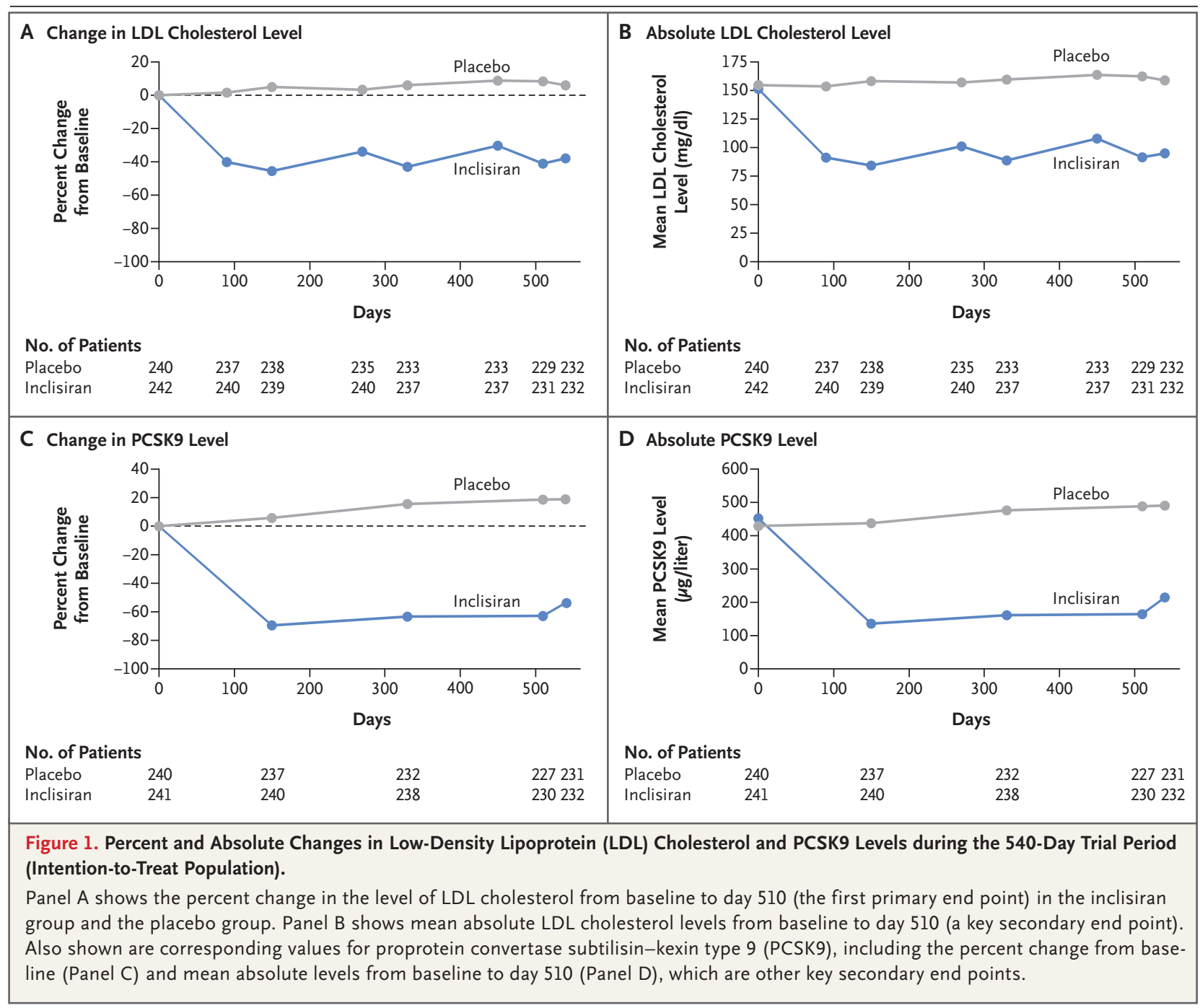

ants were found in 317 of 432 patients (73.4\%) who consented to genetic testing. Of these patients, 256 (80.8\%) had single LDLR causative variants, of whom 231 (90.2\%) had LDLR pathogenic variants, 17 (6.6\%) had probably pathogenic variants, and 8 (3.1\%) had variants that were of uncertain significance.

Of the 432 patients who were tested, 23 (5.3\%) had variants in $A P O B$, and 1 had a PCSK9 gain-offunction variant in PCSK9. In 37 patients (8.6\%), two variants were consistent with either double heterozygous familial hypercholesterolemia (a variant in LDLR and in either APOB or PCSK9), compound heterozygous disease (two different variants in LDLR), or truly homozygous disease (two identical LDLR variants). ${ }^{14,15}$ The mean baseline LDL cholesterol level in these patients was $152.4 \mathrm{mg}$ per deciliter (3.9 mmol per liter). The patients who had LDLR pathogenic variants had the highest mean baseline LDL cholesterol level (160.8 mg per deciliter [4.2 mmol per liter]).

In the inclisiran group, the mean reductions in the LDL cholesterol levels were similar in patients with LDLR pathogenic variants, probably pathogenic variants, and variants of uncertain significance. The mean between-group difference in the percent change in LDL cholesterol levels in patients with two identified variants was -41.2 percentage points. The corresponding betweengroup difference among the patients in whom a causative variant could not be identified was -59.2 percentage points; among the 50 patients 


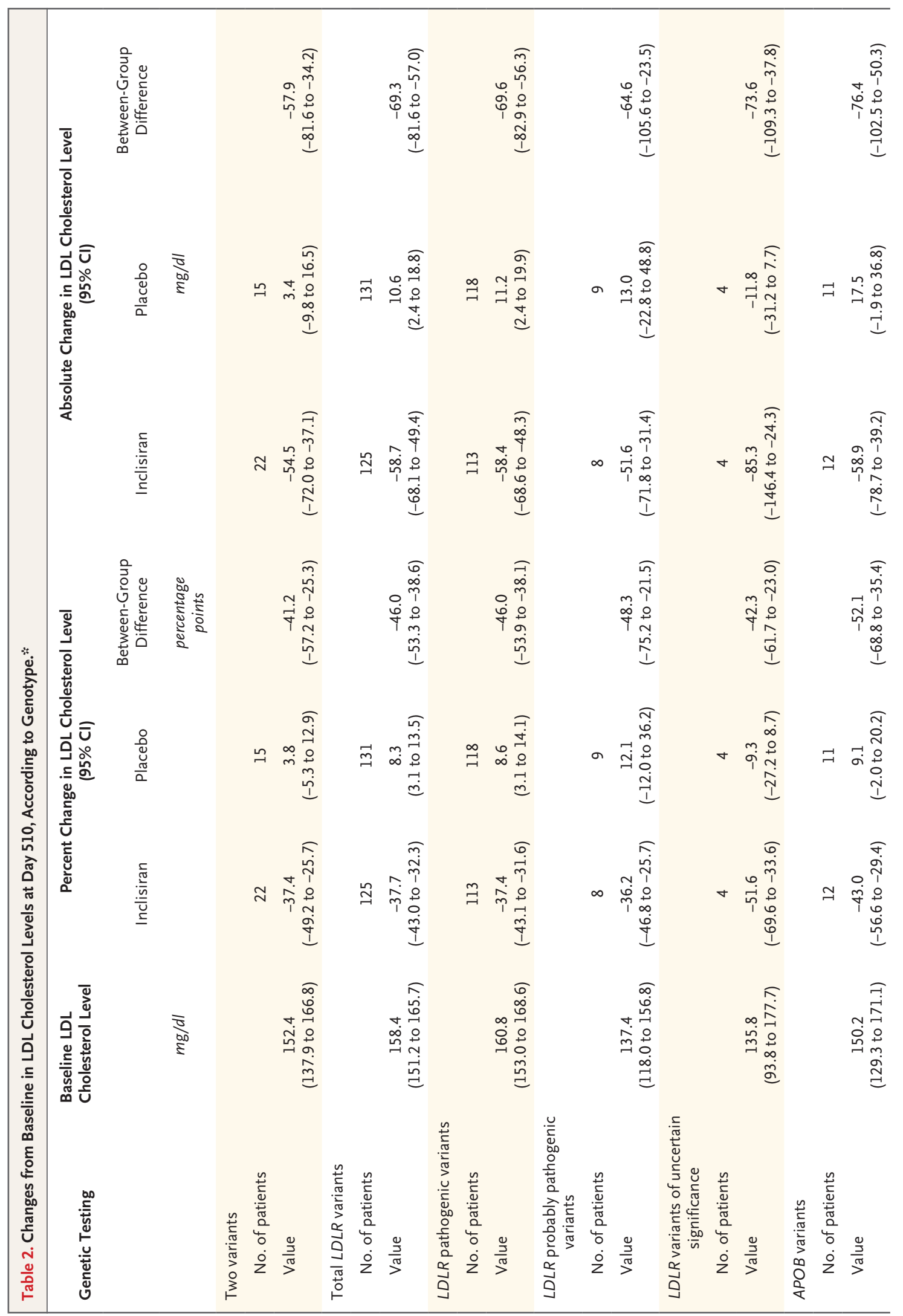




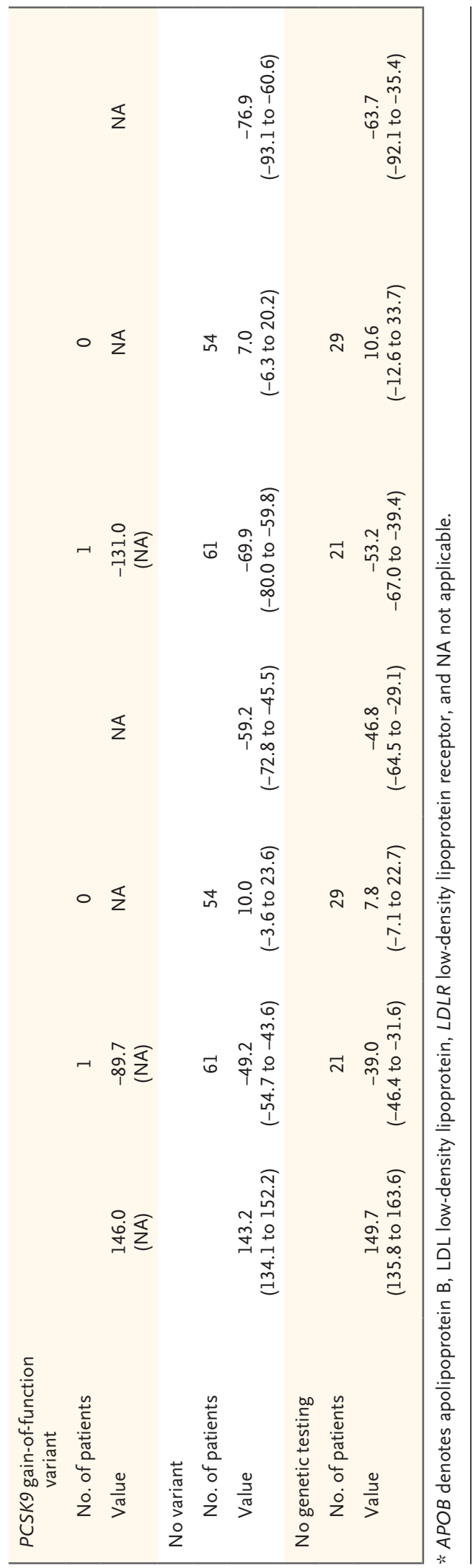

who did not undergo genetic testing, the corresponding between-group difference was -46.8 percentage points (Table 2 and Fig. S4). In patients with $A P O B$ variants in the inclisiran group, the between-group difference was -52.1 percentage points; in the single patient with a PCSK9 gain-of-function variant, the between-group difference was -89.7 percentage points.

\section{SAFETY AND ADVERSE EVENTS}

The adverse events that were reported in the safety population during treatment are shown in Table 3. Adverse events that occurred during the trial period, regardless of causality, were reported in 185 of 241 patients $(76.8 \%)$ in the inclisiran group and in 172 of 240 patients (71.7\%) in the placebo group. The majority of events $(94.6 \%$ in the inclisiran group and $91.9 \%$ in the placebo group) were reported as mild to moderate (Table S5). More patients in the inclisiran group than in the placebo group had a protocol-defined injection-site reaction $(17.0 \%$ vs. $1.7 \%)$, with the majority of events $(90.2 \%)$ graded as mild and none described as severe or persistent. There was a lower number of serious adverse events with inclisiran than with placebo (7.5\% vs. $13.8 \%$ ). These events included 1 death in each of the groups, neither of which was thought to be related to the trial intervention by the investigators.

The frequency of adverse events was similar in the two groups as assessed according to systemorgan class (Table S5). Laboratory defined adverse events were also similar between the groups (Table 3 and Table S5).

In the inclisiran group, low-titer antidrug antibodies were detected in $2.6 \%$ of the samples (25 samples from 18 patients), a finding that was consistent with assay-testing characteristics and not considered to be due to treatment with inclisiran. The presence of antidrug antibodies in posttreatment samples was often transient and not associated with changes in any pharmacologic or clinical measurements.

\section{DISCUSSION}

Heterozygous familial hypercholesterolemia is a common condition and may account for as many as 1 in 10 cases of premature acute coronary syndromes. ${ }^{16}$ The major driver of the risk of atherosclerotic cardiovascular disease among patients with this condition is the lifelong cumulative 


\begin{tabular}{|c|c|c|c|}
\hline \multirow[t]{2}{*}{ Variable } & $\begin{array}{l}\text { Inclisiran } \\
(\mathrm{N}=\mathbf{2 4 1})\end{array}$ & $\begin{array}{l}\text { Placebo } \\
(\mathrm{N}=\mathbf{2 4 0})\end{array}$ & $\begin{array}{l}\text { Risk Ratio } \\
(95 \% \mathrm{CI})\end{array}$ \\
\hline & \multicolumn{2}{|c|}{ no. of patients (\%) } & \\
\hline \multicolumn{4}{|l|}{ Adverse events } \\
\hline Patients with $\geq 1$ adverse event & $185(76.8)$ & $172(71.7)$ & $1.1(1.0-1.2)$ \\
\hline $\begin{array}{l}\text { Patients with } \geq 1 \text { adverse event leading to discontinuation of } \\
\text { trial intervention }\end{array}$ & $3(1.2)$ & 0 & NA \\
\hline \multicolumn{4}{|l|}{ Serious adverse events } \\
\hline Patients with $\geq 1$ serious adverse event & $18(7.5)$ & $33(13.8)$ & $0.5(0.3-0.9)$ \\
\hline \multicolumn{4}{|l|}{ Death } \\
\hline From any cause & $1(0.4)$ & $1(0.4)$ & $1.0(0.1-15.8)$ \\
\hline Cardiovascular cause & $1(0.4)$ & 0 & NA \\
\hline New worsening or recurrent cancer & $2(0.8)$ & $3(1.2)$ & $0.7(0.1-3.9)$ \\
\hline \multicolumn{4}{|l|}{ Other cardiovascular adverse events } \\
\hline Prespecified exploratory cardiovascular event $†$ & $10(4.1)$ & $10(4.2)$ & $1.0(0.4-2.3)$ \\
\hline Fatal or nonfatal myocardial infarction & $3(1.2)$ & $1(0.4)$ & $3.0(0.3-28.5)$ \\
\hline Fatal or nonfatal stroke & 0 & 0 & NA \\
\hline \multicolumn{4}{|l|}{ Protocol-defined injection-site reaction } \\
\hline Any event & $41(17.0)$ & $4(1.7)$ & $10.2(3.7-28.1)$ \\
\hline Mild & $37(15.4)$ & $4(1.7)$ & $9.2(3.3-25.4)$ \\
\hline Moderate & $4(1.7)$ & 0 & NA \\
\hline Severe & 0 & 0 & NA \\
\hline Persistent & 0 & 0 & NA \\
\hline \multicolumn{4}{|l|}{ Frequent adverse events } \\
\hline Nasopharyngitis & $28(11.6)$ & $20(8.3)$ & $1.4(0.8-2.4)$ \\
\hline Influenza & $13(5.4)$ & $21(8.8)$ & $0.6(0.3-1.2)$ \\
\hline Upper respiratory tract infection & $16(6.6)$ & $16(6.7)$ & $1.0(0.5-1.9)$ \\
\hline Back pain & $17(7.1)$ & $10(4.2)$ & $1.7(0.8-3.6)$ \\
\hline Injection-site reaction & $22(9.1)$ & 0 & NA \\
\hline Gastroenteritis & $11(4.6)$ & $6(2.5)$ & $1.8(0.7-4.9)$ \\
\hline \multicolumn{4}{|l|}{ Laboratory results } \\
\hline Alanine aminotransferase $>3 \times$ ULN & $3(1.2)$ & $1(0.4)$ & $3.0(0.3-28.5)$ \\
\hline Aspartate aminotransferase $>3 \times$ ULN & $2(0.8)$ & $1(0.4)$ & $2.0(0.2-21.8)$ \\
\hline Alkaline phosphatase $>3 \times$ ULN & $2(0.8)$ & 0 & NA \\
\hline Bilirubin $>2 \times$ ULN & $4(1.7)$ & $3(1.2)$ & $1.3(0.3-3.9)$ \\
\hline Creatinine $>2 \mathrm{mg} / \mathrm{dl}$ & $1(0.4)$ & $1(0.4)$ & $1.0(0.1-15.8)$ \\
\hline Creatine kinase $>5 \times$ ULN & $4(1.7)$ & $5(2.1)$ & $0.8(0.2-2.9)$ \\
\hline Platelet count $<75,000$ per $\mathrm{mm}^{3}$ & 0 & $1(0.4)$ & NA \\
\hline \multicolumn{4}{|c|}{$\begin{array}{l}\text { The safety population included all the patients who had received at least one dose of inclisiran or placebo during the } \\
\text { trial period of } 540 \text { days. One patient underwent randomization in error and therefore did not receive either inclisiran o } \\
\text { placebo. To convert the values for creatinine to micromoles per liter, multiply by } 88.4 \text {. NA denotes not applicable, and } \\
\text { ULN upper limit of the normal range. } \\
\text { Regulatory Activities as cardiac death, and any signs or symptoms of cardiac arrest, nonfatal myocardial infarction, or } \\
\text { nonfatal stroke. }\end{array}$} \\
\hline
\end{tabular}


exposure to elevated levels of LDL cholesterol, especially when there is a delay in the initiation of effective cholesterol-lowering therapy. ${ }^{17}$ In our trial, among patients who received a regimen of subcutaneous injections of inclisiran on days 1 , 90,270 , and 450 , the between-group difference in the percent change in the LDL cholesterol level from baseline to day 510 (the first primary end point) was a reduction of 47.9 percentage points in the inclisiran group. The corresponding between-group difference in the time-averaged percent change in the LDL cholesterol level between day 90 and day 540 (the second primary end point) was a reduction of 44.3 percentage points. It is notable that at baseline the majority of patients who had such reductions were receiving high-intensity statin therapy along with ezetimibe. In addition, $65 \%$ of the patients in the inclisiran group had an LDL cholesterol level of less than $100 \mathrm{mg}$ per deciliter. These reductions were achieved without additional safety signals after four injections during a 16-month period.

The robust reduction in LDL cholesterol levels in patients with different monogenic $L D L R$ variants is consistent with the findings in trials of monoclonal antibodies and suggests that the response to PCSK9 inhibition is mainly dependent on the up-regulation of normally functioning LDL receptors on the hepatocyte surface, which override the minor role of clearance of LDL cholesterol by the up-regulation of dysfunctional LDL receptors..$^{5,17,18}$ This hypothesis is also supported by the observation that patients with LDLR null homozygous familial hypercholesterolemia have either a poor response or no response to PCSK9 inhibition. ${ }^{19,20}$

The significant reductions in lipoprotein(a) levels with inclisiran, as has been seen with PCSK9 monoclonal antibody therapy, contrasts with the actions of other drugs, especially statins, which also act by up-regulating the LDL receptor. Since an elevated lipoprotein(a) level is an independent risk factor for atherosclerotic cardiovascular disease, this activity may be an additional benefit of inclisiran therapy. ${ }^{21}$

Since inclisiran acts predominantly in the liver, which is the main site of PCSK9 production, the reduction in LDL cholesterol levels with inclisiran in patients with heterozygous familial hypercholesterolemia is similar to that achieved with PCSK9 monoclonal antibody therapy. ${ }^{5,6,22,23}$ The reduction in LDL cholesterol levels of almost $50 \%$ with twice-yearly administration of inclisiran in patients with heterozygous familial hypercholesterolemia who had been receiving maximally accepted background statin therapy has the potential to improve their adherence to the treatment regimen.

Supported by the Medicines Company.

Disclosure forms provided by the authors are available with the full text of this article at NEJM.org.

A data sharing statement provided by the authors is available with the full text of this article at NEJM.org.

We thank all the patients, investigators, and staff members who participated in the trial; El Mustapha Bahassi of Medpace Reference Laboratories for performing the genotyping; and the National Institute for Health Research (NIHR) Imperial Biomedical Research Centre and the NIHR Applied Research Collaboration Northwest London for providing assistance to Dr. Ray.
REFERENCES

1. Nordestgaard BG, Chapman MJ, Humphries SE, et al. Familial hypercholesterolaemia is underdiagnosed and undertreated in the general population: guidance for clinicians to prevent coronary heart disease: consensus statement of the European Atherosclerosis Society. Eur Heart J 2013;34:3478a-90a.

2. Berberich AJ, Hegele RA. The complex molecular genetics of familial hypercholesterolaemia. Nat Rev Cardiol 2019;16: 9-20.

3. Talmud PJ, Shah S, Whittall R, et al. Use of low-density lipoprotein cholesterol gene score to distinguish patients with polygenic and monogenic familial hypercholesterolaemia: a case-control study. Lancet 2013;381:1293-301.

4. Defesche JC, Gidding SS, Harada-Shiba M, Hegele RA, Santos RD, Wierzbicki AS. Familial hypercholesterolaemia. Nat Rev Dis Primers 2017;3:17093.

\begin{abstract}
5. Raal FJ, Stein EA, Dufour R, et al. PCSK9 inhibition with evolocumab (AMG 145) in heterozygous familial hypercholesterolaemia (RUTHERFORD-2): a randomised, double-blind, placebo-controlled trial. Lancet 2015;385:331-40.

6. Kastelein JJP, Hovingh GK, Langslet G, et al. Efficacy and safety of the proprotein convertase subtilisin/kexin type 9 monoclonal antibody alirocumab vs placebo in patients with heterozygous familial hypercholesterolemia. J Clin Lipidol 2017; 11(1):195.e4-203.e4.

7. Ray KK, Landmesser U, Leiter LA, et al. Inclisiran in patients at high cardiovascular risk with elevated LDL cholesterol. N Engl J Med 2017;376:1430-40.

8. Austin MA, Hutter CM, Zimmern RL, Humphries SE. Genetic causes of monogenic heterozygous familial hypercholesterolemia: a HuGE prevalence review. Am J Epidemiol 2004;160:407-20.
\end{abstract}

9. Iacocca MA, Hegele RA. Recent advances in genetic testing for familial hypercholesterolemia. Expert Rev Mol Diagn 2017;17:641-51.

10. Iacocca MA, Wang J, Dron JS, et al. Use of next-generation sequencing to detect LDLR gene copy number variation in familial hypercholesterolemia. J Lipid Res 2017;58:2202-9.

11. Richards S, Aziz N, Bale S, et al. Standards and guidelines for the interpretation of sequence variants: a joint consensus recommendation of the American College of Medical Genetics and Genomics and the Association for Molecular Pathology. Genet Med 2015;17:405-24.

12. Chora JR, Medeiros AM, Alves AC, Bourbon M. Analysis of publicly available LDLR, APOB, and PCSK9 variants associated with familial hypercholesterolemia: application of ACMG guidelines and implications for familial hypercho- 
lesterolemia diagnosis. Genet Med 2018; 20:591-8.

13. Iacocca MA, Chora JR, Carrié A, et al. ClinVar database of global familial hypercholesterolemia-associated DNA variants. Hum Mutat 2018;39:1631-40.

14. Sjouke B, Defesche JC, Hartgers ML, et al. Double-heterozygous autosomal dominant hypercholesterolemia: clinical characterization of an underreported disease. J Clin Lipidol 2016;10:1462-9.

15. Cuchel M, Bruckert E, Ginsberg HN, et al. Homozygous familial hypercholesterolaemia: new insights and guidance for clinicians to improve detection and clinical management: a position paper from the Consensus Panel on Familial Hypercholesterolaemia of the European Atherosclerosis Society. Eur Heart J 2014;35:2146-57. 16. Amor-Salamanca A, Castillo S, Gon-
zalez-Vioque E, et al. Genetically confirmed familial hypercholesterolemia in patients with acute coronary syndrome. J Am Coll Cardiol 2017;70:1732-40.

17. Ference BA, Ginsberg HN, Graham I, et al. Low-density lipoproteins cause atherosclerotic cardiovascular disease. 1. Evidence from genetic, epidemiologic, and clinical studies: a consensus statement from the $\mathrm{Eu}-$ ropean Atherosclerosis Society Consensus Panel. Eur Heart J 2017;38:2459-72.

18. Brown MS, Goldstein JL. Lowering plasma cholesterol by raising LDL receptors. N Engl J Med 1981;305:515-7.

19. Raal FJ, Honarpour N, Blom DJ, et al. Inhibition of PCSK9 with evolocumab in homozygous familial hypercholesterolaemia (TESLA part B): a randomised, double-blind, placebo-controlled trial. Lancet 2015;385:341-50.
20. Raal FJ, Sjouke B, Hovingh GK, Isaac BF. Phenotype diversity among patients with homozygous familial hypercholesterolemia: a cohort study. Atherosclerosis 2016;248:238-44.

21. Nordestgaard BG, Chapman MJ, Ray $\mathrm{K}$, et al. Lipoprotein(a) as a cardiovascular risk factor: current status. Eur Heart J 2010;31:2844-53.

22. Fitzgerald K, White S, Borodovsky A, et al. A highly durable RNAi therapeutic inhibitor of PCSK9. N Engl J Med 2017; 376:41-51.

23. Nair JK, Willoughby JLS, Chan A, et al. Multivalent N-acetylgalactosamineconjugated siRNA localizes in hepatocytes and elicits robust RNAi-mediated gene silencing. J Am Chem Soc 2014;136: 16958-61.

Copyright $(2020$ Massachusetts Medical Society. 